Results Using research examples (domestic ladders, motorised mobility scooters and fire risk reduced cigarettes), the problem will be summarised by investigation of the adequacy of existing data systems to identify injuries associated with these existing and emerging products; the effects of jurisdictional boundaries on product safety; the reactive nature of the system and related limitations; and the lack of an adequate scientific evidence base to proactively guide much of the related regulatory process (e.g. Product Safety Standards).

Conclusions Potential new multi-sector approaches to developing a more proactive product safety system that aligns evidence with public policy and the regulatory system will be proposed.

\section{RE:FINE NEISS: A REAL-TIME INTERACTION SEARCH SYSTEM FOR CONSUMER PRODUCT-RELATED INJURY ED VISITS IN UNITED STATES}

${ }^{1}$ Soheil Moosavinasab, ${ }^{1}$ Jeremy Patterson, ${ }^{2}$ Krista K Wheeler, ${ }^{1}$ Robert Strouse, ${ }^{2}$ Huiyun Xiang, ${ }^{1}$ Yungui Huang, ${ }^{1}$ Simon M Lin. ${ }^{1}$ Research Information Solutions and Innovation And; ${ }^{2}$ Centre for Paediatric Trauma Research, the Research Institute at Nationwide Children's Hospital, Columbus, Ohio, USA

\subsection{6/injuryprev-2016-042156.352}

Background The United States Consumer Product Safety Commission's (CPSC) National Electronic Injury Surveillance System (NEISS) records information about consumer product-related injuries from a probability sample of emergency departments, and makes these records publicly available. NEISS is used for injury research and to guide decisions about product recall, set product safety standards, and develop public awareness campaigns. The NEISS data contain both structured and unstructured fields. However, the current NEISS query builder system only allows structured data query.

Methods We redesigned the online data query system and created RE:fine NEISS to enable both structured and unstructured data query while enhancing the user experience. Our design goals were to provide real-time feedback, reduce the cognitive load for users and offer advanced functionalities.

Results The RE:fine NEISS significantly improves the usability of the NEISS. As the user builds a query, a sample of the matching subset of data is populated in real-time and is updated each time the user modifies the query. The auto-complete lookup feature for product names makes finding products easier. In addition, the unstructured text search capability allows users to add as many conditions as they want for any field search for keywords, phrases and wildcards, apply nested Boolean queries, and exclude conditions. Furthermore users can save, share, and reuse their queries.

Conclusions A group of injury researchers, familiar with NEISS, assisted with the development and evaluation of RE:fine NEISS. These users found that they could easily generate queries, readily view the data in a friendly format, quickly assess project feasibility, and produce the desired dataset for more detailed study. RE: fine NEISS is available online at http://injurysearch.nationwidechildrens.org/

\section{PRODUCT RELATED HEAD INJURIES AMONG INFANTS AND TODDLERS IN EUROPE - A PUBLIC HEALTH TOPIC?}

${ }^{1}$ Gabriele Ellsaesser, ${ }^{1}$ Frank Gries, ${ }^{2}$ Samantha Turner, ${ }^{2,3}$ Ronan A Lyons, ${ }^{4}$ Bjarne Larsen, ${ }^{5}$ Wim Rogmans, ${ }^{6}$ Rupert Kisser, ${ }^{7}$ Huib Valkenberg, ${ }^{8}$ Dritan Bejko, ${ }^{9}$ Monica Steiner, ${ }^{9}$ Robert Bauer. 'Brandenburg State Office of Environment, Health and Consumer Protection, Germany; ${ }^{2}$ Farr Institute Swansea, University, Medical School, UK; ${ }^{3}$ Public Health Wales NHS Trust, UK; ${ }^{4}$ National Institute of Public Health, Denmark; ${ }^{5}$ Eurosafe, The Netherlands; ${ }^{6}$ Eurosafe, Austria; ${ }^{7}$ Consumer Safety Institute, The Netherlands; ${ }^{8}$ Centre d'Etudes en Santé Publique, Luxembourg; ${ }^{9}$ Austrian Road Safety Board, Austria

\subsection{6/injuryprev-2016-042156.353}

Background Studies show that young children $(<5$ yrs $)$ are most at risk among the under 18-year-olds for being hospitalised because of a head injury (Dunning et al. 2004). Despite the high incidence rates in this age group and some publications on a national level (Ellsaesser 2014), little knowledge exists on a European level of the importance of products triggering head injuries in young children. For such questions the European Injury Data Base (IDB) provides a valuable source of information, in particular its full data set (FDS) which is used in $18 \mathrm{EU}$ countries for collecting information on the product involvement. The following study aims to use this data for an in depth analysis on product related head injuries.

Method Case analysis of a total of 54,001 injuries collected during a 2-year period (2013-2014) in the under five-year-olds treated in 115 hospitals (either ward or emergency) of 18 European countries. An injury was counted as "head injury" if the body part was documented as head and one of the following injury types were registered: contusion, open wound, abrasion, fracture, concussion or other specified brain injury. An injury has been counted as a product related injury if a product was registered as triggering the injury.

Results Head injuries in infants ( $<1$ year) made up 63\% $(3,486)$ of all injuries $(5,538)$ in the age group. $65 \%(2,255)$ of head injuries were triggered by products. The three most frequent product related head injuries were falling from or out of: \#1 bed $20 \%$ (459), \#2 changing table 10\% (226), \#3 buggy or carrier 7\% (150). Head injuries in 1-to 4-year-olds made up 41\% $(19,876)$ of all injuries in the age group $(48,463) .59 \%(6,977)$ were triggered by a product. The three most frequent products involved were: \#1 furniture 8\% (1048), e.g. couch, \#2 stairs 7\% (963), \#3 bed 5\% (653).

Conclusions Product related head injuries in young children are a crucial public health issue and new parents should be given targeted injury prevention measures.

\section{DEVELOPING RISK-INFORMED DECISION-MAKING PROCESSES}

Minna Päivinen. Finnish Safety and Chemicals Agency (Tukes), Finland

\subsection{6/injuryprev-2016-042156.354}

Background Finnish Safety and Chemicals Agency (Tukes) is a national agency which supervises and promotes technical safety and conformity, together with consumer and chemicals safety. One of the key tasks of Tukes is to promote safety awareness and behaviour as well as to ensure that all participants abide by the legislation.

Current changes in society and technology are extremely rapid and the variety of products on the market is ever increasing. At the same time the authorities' resources are continuing to 
decrease. This creates a need to develop new approaches for surveillance.

As a solution, Tukes is directing its actions and prioritizations towards more risk-informed decision-making (RIDM). The aim is to find methods for efficient implementation of risk-informed prioritisation and thus new ways to promote safety. RIDM can be applied in the selection of the targets and/or the methods of the operations. The challenge is to create efficient and reliable processes for this.

Methods Tukes' main processes are surveillance and enforcement, communications and development. RIDM can be applied to all of these, but the areas require different approaches. Currently Tukes is developing and testing methods especially for surveillance and enforcement.

Results The development work is in progress and results will be ready to be presented in Safety 2016 conference.

Conclusions As the safety field is getting more complex and at the same time available resources for safety promotion and surveillance are decreasing, new ways to develop safety are needed. RIDM allows Tukes to develop more efficient ways to promote safety and to direct the actions to the areas which have the most or highest risks.

\section{Disabled Persons and Safety}

\section{Parallel Tue 3.6}

\section{THE RISK OF INJURIES IN CHILDREN WITH ATTENTION DEFICIT-HYPERACTIVITY DISORDER (ADHD) IN ENGLAND}

Vibhore Prasad, Kapil Sayal, Joe West, Denise Kendrick. University of Nottingham, UK

\subsection{6/injuryprev-2016-042156.355}

Background Attention deficit-hyperactivity disorder (ADHD) is a common in children with a prevalence of 5\%. Injuries are common and are a leading cause of morbidity and mortality in England and worldwide. ADHD may be associated with an increased risk of injuries but accurate and detailed risk estimates are lacking.

Methods A cohort study was conducted using primary care and hospital records from the Clinical Practice Research Datalink (CPRD) (1998 to 2012). All children with ADHD (aged 3 to 17 years) were frequency-matched, by age band, to up to twenty children without ADHD. Hazard ratios for (long bone) fractures, thermal injuries and poisonings were estimated using Cox regression and adjusted for age, sex, strategic health authority region, area-level deprivation quintile (measured using Index of Multiple Deprivation) and the calendar year when the child entered the study.

Results There were 15,737 children with and 291,894 without ADHD. 84.6\% with ADHD were boys compared to 50.7\% without ADHD. The adjusted risk of fractures in children with ADHD was $28 \%$ greater than in children without $(\mathrm{HR}=1.28$ (1.22 to 1.35$)$ ). The adjusted risk of thermal injuries in children with ADHD was more than double the risk in children without $(\mathrm{HR}=2.04$ (1.80 to 2.31$))$. The adjusted risk of poisonings was nearly 4 times greater in children with ADHD compared to children without $(\mathrm{HR}=3.99$ (3.58 to 4.44$))$. The adjusted risk of long bone fractures was similar to the risk of any fractures $(\mathrm{HR}=1.23$ (1.16 to 1.31$)$ ).

Conclusions Children with ADHD are at a greater risk of fractures, thermal injuries and poisonings than children without. All services involved in the care and education of children with ADHD should be aware of the risks. The estimated risks of injury should be communicated to children and their parents at the time of diagnosis, medication reviews and follow-up visits.

\section{IS ADHD ASSOCIATED WITH UNINTENTIONAL INJURIES AMONG CHILDREN? A MATCHED CASE CONTROL STUDY PAKISTAN}

${ }^{1}$ Hasana Qadri, 'Zafar Fatmi, ${ }^{1}$ Shafquat Rozi, ${ }^{2}$ Sanober Nadeem, ${ }^{3}$ Nadeemullah Khan, ${ }^{3}$ Uzma Rahim Khan. 'Department of Community Health Sciences, the Aga Khan University, Pakistan; '2Department of Early Childhood Development, the Aga Khan University, Pakistan; ${ }^{3}$ Department of Emergency Medicine, the Aga Khan University, Pakistan

\subsection{6/injuryprev-2016-042156.356}

Background ADHD and unintentional injuries are common among young children. However, epidemiological data investigating association between $\mathrm{ADHD}$ and unintentional injuries is scarce, both from developed and developing countries.

Method A hospital based case control study was conducted. A total of 300 children, 4-11 years of age with unintentional injuries (case) and 600 children with health conditions other than unintentional injuries (two controls for each case), matched for age and gender, were recruited from the emergency room (ER) of three tertiary care hospitals of Karachi: on each belonging to public and private sector. Unintentional injuries (cases) were falls, burns, drowning, poisoning and road traffic injuries, as defined by the consulting physician in the ER. Children with complaints and diagnosis other than injuries (controls) were selected from the same hospital ER within a week of case recruitment. ADHD was defined by administering strength and difficulty questionnaire (SDQ), a validated instrument for screening of ADHD, to the primary care givers of the children (parents or guardians). Conditional logistic regression was conducted to determine association of ADHD with unintentional injuries, adjusting for potential confounders.

Results Children with ADHD were twice likely $(\mathrm{mOR}=2.2$, 95\% CI: $1.5-3.0)$ to have unintentional injury. In addition, children with low socio-economic status ( $\mathrm{mOR}=1.7,95 \% \mathrm{CI}: 1.1$ $2.4)$, more than two siblings ( $\mathrm{mOR}=2.1,95 \% \mathrm{CI}: 1.1-4.4)$, low mother education $(\mathrm{mOR}=2.0,95 \% \mathrm{CI}: 1.3-3.0)$ and history of previous injuries $(\mathrm{mOR}=1.9,95 \% \mathrm{CI}: 1.4-2.7)$ were independently related to unintentional injuries.

Conclusion Screening of ADHD among young age children along with parental counselling for the prevention of injuries would help in decreasing the disease burden. Prevention plans focusing on low socio-economic background children and with children of mother with low education may further reduce the incidence of unintentional injuries. 